\title{
Apresentação atípica de metástase de adenocarcinoma gástrico: relato de caso
}

\author{
Atypical presentation of gastric adenocarcinoma metastasis: case report \\ Presentación atípica de metástasis de adenocarcinoma gástrico: reporte de un caso
}

Recebido: 22/09/2021 | Revisado: 26/09/2021 | Aceito: 27/09/2021 | Publicado: 30/09/2021

Maria Clara Faustino Linhares

ORCID: https://orcid.org/0000-0002-2932-8929 Hospital Hélio Angotti, Brasil

E-mail: mclaralinhares@ hotmail.com

Maria Nathália Vilela Assis

ORCID: https://orcid.org/0000-0003-1491-5642 Hospital Hélio Angotti, Brasil

E-mail: maria.nathaliaa@hotmail.com

Carolina Bugiato Faria Salge

ORCID: https://orcid.org/0000-0001-9692-0829 Hospital Hélio Angotti,Brasil

E-mail: carol_salge_@hotmail.com

Fernanda Dias de Paula

ORCID: https://orcid.org/0000-0002-1728-966X

Universidade de Uberaba, Brasil

E-mail: fernandadiasddp@gmail.com

Guilherme Nascentes Schmitt

ORCID: https://orcid.org/0000-0001-7697-6825 Hospital Hélio Angotti, Brasil

E-mail: guilhermeschmitt96@ hotmail.com

Heloísa Paula Yamaguti

ORCID: https://orcid.org/0000-0002-9704-5568

Universidade Federal do Triângulo Mineiro, Brasil

E-mail: heloisapy@hotmail.com

Vitor Antônio Santos Anjo

ORCID: https://orcid.org/0000-0002-3880-865X

Hospital Hélio Angotti, Brasil

E-mail: vitor-anjo@hotmail.com

Guilherme Freire Angotti Carrara

ORCID: https://orcid.org/0000-0001-8506-481X Hospital Hélio Angotti, Brasil E-mail: gcarrara@yahoo.com

Luiz Carlos Furtado de Almeida Junior

ORCID: https://orcid.org/0000-0002-1388-2829 Hospital Hélio Angotti, Brasil

E-mail: luizcarlos.med@gmail.com

\begin{abstract}
Resumo
Introdução: O câncer gástrico é o $5^{\circ}$ câncer mais incidente, excetuando-se tumores de pele não melanoma e a $3^{\text {a }}$ causa de morte decorrente de câncer no mundo. Os locais mais comuns de metástases do câncer gástrico são: fígado (48\%), peritônio (32\%), pulmão (15\%) e osso (12\%). Objetivo: apresentar o curso de metástase cutânea em decorrência de um adenocarcinoma gástrico. Metodologia: Estudo descritivo do tipo relato de caso, cujos dados foram obtidos no prontuário físico da paciente. Apresentação do caso: Reportamos o caso de uma paciente do sexo feminino, 44 anos, submetida a tratamento cirúrgico e quimioterápico devido a um adenocarcinoma gástrico. Um ano e quatro meses após a intervenção inicial, observou-se o aparecimento de lesões cutâneas hipercrômicas, dolorosas, difusas e não pruriginosas, localizadas principalmente em tórax, dorso e membros superiores. A biópsia cutânea confirmou a hipótese de metástase cutânea de adenocarcinoma gástrico. Conclusão: No contexto do tratamento do câncer gástrico, faz-se importante a vigilância de novos sinais e sintomas, como lesões cutâneas insidiosas, aventando a possibilidade de tumor cutâneo metastático.
\end{abstract}

Palavras-chave: Câncer gástrico; Metástase; Adenocarcinomas.

\section{Abstract}

Introduction: Gastric cancer is the 5th most incident cancer, except for non-melanoma skin tumors and the 3rd leading cause of cancer death in the world. The most common sites of gastric cancer metastases are: liver (48\%), peritoneum (32\%), lung (15\%) and bone (12\%). Objective: to present the course of cutaneous metastasis due to gastric adenocarcinoma. Methodology: Descriptive study of the case report type, whose data were obtained from the patient's 
medical record. Case presentation: We report the case of a 44-year-old female patient who underwent surgical and chemotherapy treatment due to gastric adenocarcinoma. One year and four months after the initial intervention, hyperchromic, painful, diffuse and non-pruritic skin lesions appeared, located mainly on the chest, back and upper limbs. Skin biopsy confirmed the hypothesis of cutaneous metastasis from gastric adenocarcinoma. Conclu sion: In the context of gastric cancer treatment, it is important to monitor new signs and symptoms, such as insidious skin lesions, suggesting the possibility of metastatic skin tumor.

Keywords: Gastric cancer; Metastasis; Adenocarcinomas.

\section{Resumen}

Introducción: El cáncer gástrico es el quinto cáncer más comun, a excepción de los tumores de piel no melanoma y la tercera causa principal de muerte por cáncer en el mundo. Los sitios más comunes de metástasis de cáncer gástrico son: hígado (48\%), peritoneo (32\%), pulmón (15\%) y hueso (12\%). Objetivo: presentar el curso de la metástasis cutánea por adenocarcinoma gástrico. Metodología: Estudio descriptivo del tipo de reporte de caso, cuyos datos se obtuvieron de la historia clínica del paciente. Presentación del caso: Presentamos el caso de una paciente de 44 años que se sometió a tratamiento quirúrgico y quimioterápico por un adenocarcinoma gástrico. Un año y cuatro meses después de la intervención inicial aparecieron lesiones cutáneas hipercrómicas, dolorosas, difusas y no pruriginosas, localizadas principalmente en tórax, espalda y miembros superiores. La biopsia de piel confirmó la hipótesis de metástasis cutánea de adenocarcinoma gástrico. Conclusión: En el contexto del tratamiento del cáncer gástrico, es importante monitorear nuevos signos y síntomas, como lesiones cutáneas insidiosas, que sugieren la posibilidad de un tumor cutáneo metastásico.

Palabras clave: Cáncer gástrico; Metástasis; Adenocarcinomas.

\section{Introdução}

O câncer gástrico (CG) é o $5^{\circ}$ câncer mais incidente, excetuando-se tumores de pele não melanoma e a $3^{\mathrm{a}}$ causa de morte decorrente de câncer no mundo (Sung et al, 2021). Mundialmente, a incidência de CG tem se mostrado mais comum em homens e eleva-se com a idade, sendo a maioria dos casos após os 60 anos (Ferlay et al, 2015). O adenocarcinoma é o tipo histológico mais frequente, correspondendo a mais de 95\% de todos os cânceres gástricos. (Asplund J, 2018). Segundo Marqués- Lespier e Colaboradores (2016), o prognóstico do CG é em grande parte dependente do estágio do tumor no momento do diagnóstico.

O CG é uma comorbidade multifatorial, na qual diversos fatores podem influenciar seu desenvolvimento, tanto ambientais, quanto genéticos (Machloska et al, 2020). Os fatores de risco imutáveis do CG incluem sexo, idade, história familiar e raça. Os fatores preveníveis são tabagismo, radiação, infecção por Helicobacter pylori, alta ingestão de sal e baixa ingestão de frutas e vegetais. (Smyth et al, 2020).

Metástases podem ser fatais, causando efeito de massa, ou até mesmo, levando à falha na homeostase. Nas últimas duas décadas, houve aumento em mais de $40 \%$ da proporção de portadores de câncer gástrico com metástases. Os locais mais comuns são: fígado (48\%), peritônio (32\%), pulmão (15\%) e osso (12\%). (Riihimäki M, 2016). Todavia, segundo Gündüz e Colaboradores (2017), os cânceres de pele metastáticos são doenças malignas dermatológicas raras, constituindo $2 \%$ de todos os tumores de pele, e estes podem ser de origem gástrica.

Desta forma, o presente relato de caso visa apresentar o curso de metástase cutânea em decorrência de um adenocarcinoma gástrico.

\section{Metodologia}

\section{Desenho do Estudo}

Trata-se de um estudo descritivo do tipo relato de caso, utilizando-se dados obtidos no prontuário físico da paciente. Para realizar o estudo de caso, foi feito assim como Pereira A. S. et al. (2018), analisando a existência de algum fenômeno que tornasse o estudo interessante para a sociedade e identificando quais características tornavam o estudo um caso. 


\section{Local do estudo}

O Hospital Hélio Angotti (HHA) é uma entidade privada sem fins lucrativos, inaugurado no ano de 1961 e localizado na cidade de Uberaba - Minas Gerais (MG), cuja finalidade é prestar assistência oncológica à sociedade.

O HHA está inserido na Rede de Atenção Especializada da Macrorregião de Saúde Triângulo do Sul, composta por 27 municípios, atendendo mais de 800 mil habitantes.

É considerado referência nacional no atendimento de alta complexidade, principalmente na prevenção, diagnóstico e tratamento oncológico.

\section{População alvo}

Um único paciente, do sexo feminino, com diagnóstico de metástase cutânea de adenocarcinoma gástrico.

\section{Técnica e instrumento de coleta de dados}

Análise do prontuário para descrição do caso clínico e revisão bibliográfica utilizando as bases científicas de dados.

\section{Aspectos Éticos}

O presente relato de caso foi aprovado pelo Comitê de Ética e Pesquisa com Seres Humanos da Faculdade de Talentos Humanos, sob o número de registro CAAE 51964421.0.0000.9028 e Parecer Consubstanciado número 109594/2021.

\section{Resultados}

AMF, feminino, 44 anos, com quadro clínico de epigastralgia, foi diagnosticada com adenocarcinoma gástrico, do tipo difuso e pouco diferenciado com metástase locorregional para linfonodos (T3N2M0).

Inicialmente foi realizada neoadjuvância com quatro ciclos de quimioterapia com Fluorouracil, Leucovorin, Oxaliplatina e Taxano (FLOT) e posteriormente, realizada gastrectomia total com linfadenectomia a D2 e reconstrução em Y de Roux, seguida de adjuvância com mais seis ciclos de FLOT.

A paciente não possuía fatores de risco conhecidos, como tabagismo, alcoolismo, infecção pela bactéria Helicobater pylori, dieta rica em defumados e nitrogenados e história familiar.

Foi realizado seguimento ambulatorial e um ano após o procedimento cirúrgico apresentou um quadro de dor abdominal associada a constipação intestinal, identificando-se oclusão ao toque retal. Foi submetida a uma laparotomia exploratória, na qual foi visualizada carcinomatose peritoneal, optando-se, portanto, em realizar apenas uma colostomia em alça intestinal.

Após o diagnóstico de metástase peritoneal, foi iniciado novo ciclo de quimioterapia com Antraciclina (Xeloda), o qual foi mantido por dois meses.

Um ano e quatro meses após a intervenção inicial, a paciente relatou o aparecimento de lesões cutâneas hipercrômicas, dolorosas, difusas e não pruriginosas, localizadas principalmente em tórax, dorso e membros superiores (Figura 1A e 1B). Foi realizada biópsia cutânea, com laudo anatomopatológico confirmando a hipótese de metástase cutânea de adenocarcinoma gástrico. 
Figura 1: Representação dos locais de surgimento das lesões cutâneas.



Fonte: Autores (2021).

Após o diagnóstico de metástase cutânea, o ciclo de quimioterapia com Antraciclina foi interrompido e substituído por Paclitaxel associado a Ramucirumab.

Um mês após o início dos novos quimioterápicos, houve redução das lesões cutâneas, com previsão de manutenção da quimioterapia por, aproximadamente, 12 ciclos, a depender da resposta clínica apresentada pela paciente durante acompanhamento mensal.

\section{Discussão}

As metástases cutâneas provenientes de neoplasias viscerais são relativamente raras, com uma taxa de incidência de cerca de 5\% (Krathen; Orengo; Rosen; 2003), de modo que essas metástases configuram cerca de $2 \%$ do total de cânceres de pele registrados (Gündüz et al, 2017). Quando presentes, o local mais comum de metástase cutânea é a parede abdominal, onde é denominado nódulo da Irmã Mary Joseph. Menos comumente, há relatos em couro cabeludo, pálpebras, pontas dos dedos, pescoço e tronco (Ghosh et al, 2021).

Os principais sítios primários que originam metástases cutâneas são câncer de mama, pulmão, cólon, cavidade oral, rim ou ovário (Koyama et al, 2019). O CG raramente se relaciona com metástases cutâneas, mais comumente observam-se sítios metastáticos em fígado, cavidade peritoneal e linfonodos (Cesaretti et al, 2014). Dentre todas as metástases cutâneas originárias do CG, os percentuais variam de $6 \%$ nos homens a $1 \%$ nas mulheres (Ghosh et al, 2021).

Comumente, as manifestações cutâneas se apresentam tardiamente, como visto no presente relato, mas também evidenciado por outros pesquisadores (Cokgezer et al., 2020; Rakka et al., 2019; Yang et al., 2020). De acordo com Rakka et al (2019), as metástases cutâneas de CG são inoperáveis e estão associadas a um estágio avançado da doença. Corroborando com esse ponto de vista, Kirchberger (2018), relata que a manifestação subcutânea e cutânea de CG é muito rara e está associada a um mau prognóstico e a uma doença metastática generalizada. No entanto, segundo Cesaretti e Cols (2014), as metástases cutâneas podem ser o primeiro sinal de uma neoplasia maligna visceral.

Similarmente ao caso apresentado no presente trabalho, Yang et al (2020), reporta um caso em que um paciente de 57 anos submetido a gastrectomia distal com linfadenectomia D2 e reconstrução em Billroth II associado a quimioterapia adjuvante, devido a um adenocarcinoma pouco diferenciado com células em anel de sinete. O estadiamento patológico foi IIIA (T3N2M0), apresentando apenas invasão vascular. O paciente apresentava história familiar positiva para câncer gástrico, mas assim como no presente relato, não apresentava outros fatores de risco.

Ainda, segundo relato de Yang e Cols (2020), após dois anos e meio da intervenção cirúrgica, o paciente iniciou com quadro de cervicalgia, sendo encontrado ao exame físico um espessamento irregular, de aproximadamente 10x12 centímetros, 
do tecido celular subcutâneo na região cervical e escapular bilateralmente. No estudo anatomopatológico, por meio da imunohistoquímica das lesões subcutâneas, foi possível evidenciar que o tecido era originado do adenocarcinoma primário metastático. Optou-se por iniciar novo esquema quimioterápico com Docetaxel, Cisplatina e 5-Fluorouracil, obtendo remissão das lesões após início da terapia.

De acordo com estudo de caso apresentado por Cokgezer et al (2020), em que uma paciente de 75 anos previamente submetida a gastrectomia total associada à linfadenectomia D2 associada a quimioterapia e radioterapia adjuvantes, devido a um adenocarcinoma gástrico de células em anel de sinete, observou-se o aparecimento de uma lesão de aspecto cicatricial em região epigástrica após três anos do diagnóstico inicial, a qual foi submetida a biópsia e confirmada a presença de células em anel de sinete, permitindo fechar o diagnóstico para carcinoma metastático de células em anel de sinete. Para o tratamento metastático, foi iniciado novo ciclo de quimioterapia com 5-fluorouracil e oxaliplatina.

Em um estudo conduzido por Namikawa et al (2017), foi relatado o caso de um paciente de 59 anos que iniciou com quadro de dor no ombro esquerdo. Ao exame físico, foi observada uma lesão nodular dolorosa em hemitórax direito, de aproximadamente dois centímetros de diâmetro, foi realizada ressecção da massa localizada em parede torácica, cujo anatomopatológico confirmou a presença de células de adenocarcinoma indiferenciado. $O$ paciente foi submetido a quimioterapia adjuvante com oxaliplatina e fluoropirimidina oral, entretanto, veio a óbito seis meses após o diagnóstico devido ao comprometimento hepático e peritoneal da doença.

Atualmente, os métodos de tratamento para CG incluem cirurgia, radioterapia, quimioterapia e imunoterapia (Tan Z, 2019). Segundo, Chen e Colaboradores (2021), a radioterapia e a quimioterapia podem reduzir a mortalidade em pacientes com CG avançado que não tolerariam a cirurgia ou para os quais a cirurgia provavelmente não traria benefícios.

No que tange à condução terapêutica dos casos de metástases cutâneas, não há dentre os estudos apresentados uma homogeneidade, visto que existiram estudos com abordagem quimioterápica exclusiva, como realizado no presente estudo (Cokgezer et al., 2020; Yang et al., 2020), mas também houve reporte da associação de quimioterapia com a abordagem cirúrgica (Namikawa et al., 2017).

Outro aspecto a destacar entre o presente relato e os demais estudos da literatura, está na idade de apresentação da lesão cutânea, visto que a paciente relatada se encontra na quinta década de vida, e nos demais estudos todos os pacientes estavam acima da sexta década de vida. Todavia, não há na literatura reporte acerca da média de idade para o surgimento de metástase cutânea de origem gástrica.

\section{Considerações Finais}

No contexto do tratamento do câncer gástrico, faz-se importante a vigilância de novos sinais e sintomas, como lesões cutâneas insidiosas, aventando a possibilidade de tumor cutâneo metastático.

O presente relato tem como finalidade compartilhar experiência clínica com outros profissionais de saúde, permitindo melhor diagnóstico e abordagem terapêutica de quadros clínicos semelhantes.

Portanto, em vista da diminuta base científica sobre o tema, sugere-se que mais estudos acerca das lesões cutâneas de origem metastática de adenocarcinomas gástricos sejam realizados e reportados na literatura.

\section{Referências}

Asplund, J., Kauppila, J. H., Mattsson, F. \& Lagergren, J. (2018). Survival Trends in Gastric Adenocarcinoma: A Population-Based Study in Sweden. Ann Surg Oncol. 25 (9): 2693-702

Cesaretti, M., Malerba, M., Basso, V., Boccardo, C., Santoni, R., D'Alessandro, G., Weiss, A., Campisi, C. \& De Cian, F. (2014). Cutaneous metastasis from primary gastric cancer: a case report and review of the literature. Cutis. 93(4):E9-E13. 
Chen, Y., Yang, Y. C., Tang, L. Y., Ge, Q. M., Shi, W. Q., Su, T., Shu, H. Y., Pan, Y. C., Liang, R. B., Li, Q. Y., \& Shao, Y. (2021). Risk Factors and Their Diagnostic Values for Ocular Metastases in Gastric Adenocarcinoma. Cancer management and research, 13, 5835-5843.

Cokgezer, S., Samanci, N. S., Bektas, M., Kepil, N. \& Demirelli, F. H. (2020). Cutaneous Metastasis of Signet Cell Gastric Carcinoma. Indian J Dermatol. 65(2):148-50.

Ferlay, J., Soerjomataram, I., Dikshit, R., Eser, S., Mathers, C., Rebelo, M., Parkin, D. M., Forman, D. \& Bray, F. (2015). Cancer incidence and mortality worldwide: sources, methods and major patterns in GLOBOCAN 2012. International journal of cancer, 136(5), E359-E386.

Ghosh, J., Arun, I., Ganguly, A. \& Ganguly. S. Cutaneous metastases in a patient with adenocarcinoma of the stomach. Indian J Dermatol Venereol Leprol 2021;87:699-701.

Gündüz, Ö., Emeksiz, M.C., Atasoy, P., Kidir, M., Yalçin, S. \& Demirkan, S. (2017) Signet-ring Cells in the Skin: A Case of Late-onset Cutaneous Metastasis of Gastric Carcinoma and a Brief Review of Histological Approach. Dermatol Reports, 8.

Kirchberger, M. C. (2018). Unusual presentation of a cutaneous metastasis in the face arising from gastric cancer: a case report. SAGE Open Medical Case Reports.

Krathen, R. A., Orengo, I. F. \& Rosen, T. (2003). Cutaneous metastasis: a meta-analysis of data. Southern medical journal, 96(2), $164-167$.

Koyama, R., Maeda, Y., Minagawa, N., Shinohara, T. \& Hamada, T. (2019) Late Cutaneous Metastasis Originating from Gastric Cancer with Synchronous Metastasis. Case Rep Gastroenterol. 26;13(1):95-101.

Machlowska, J., Baj, J., Sitarz, M., Maciejewski, R. \& Sitarz, R. (2020) Gastric Cancer: Epidemiology, Risk Factors, Classification, Genomic Characteristics and Treatment Strategies. Int. J. Mol. Sci., 21, 4012.

Marqués-Lespier, J. M., González-Pons, M. \& Cruz-Correa, M. (2016). Current Perspectives on Gastric Cancer. Gastroenterology clinics of North America, $45(3), 413-428$.

Namikawa, T., Munekage, E., Munekage, M., Maeda, H., Yatabe, T., Kitagawa, H., Kobayashi, M. \& Hanazaki, K. (2017) Subcutaneous metastasis arising from gastric cancer: A case report. Mol Clin Oncol.6(4):515-516.

Pereira A. S. et al. (2018). Metodologia da pesquisa científica. [free e-book]. Ed. UAB/NTE/UFSM.

Rakka, M., Haidar, A., Bazzi, N., Safadi, A.E. \& Chahine, G. (2019). Metachronous Skin Metastasis of Gastric Cancer: Case Report. Juniper Online Journal of Case Studies, Juniper Publishers Inc., vol. 10(4), pages 74-77.

Riihimäki, M., Hemminki, A., Sundquist, K., Sundquist, J. \& Hemminki, K. (2016). Propagação metastática em pacientes com câncer gástrico. Oncotarget. 7 (32): 52307-52316.

Smyth, E. C., Nilsson, M., Grabsch, H. I., van Grieken, N. C. \& Lordick, F. (2020). Gastric cancer. Lancet (London, England), $396(10251)$, $635-648$.

Sung, H., Ferlay, J., Siegel, R.L., Laversanne, M., Soerjomataram, I., Jemal, A. \& Bray, F. (2021). Global Cancer Statistics 2020: GLOBOCAN Estimates of Incidence and Mortality Worldwide for 36 Cancers in 185 Countries. CA Cancer J Clin. 71(3):209-249.

Tan, Z. (2019). Recent Advances in the Surgical Treatment of Advanced Gastric Cancer: A Review. Medical science monitor : international medical journal of experimental and clinical research, 25, 3537-354.

Yang, S., Liu, X.L., Guo, X.L., Song, B., Li, S.Z., Sun, X.F. \& Feng, Y. (2020) Solitary metastasis to the skin and colon from gastric cancer after curative gastrectomy and chemotherapy: A case report. Medicine (Baltimore). 31;99(31):e21532. 